\title{
Simulation et théorie : des rapports ambigus
}

À lire certains journaux de physique, il semble que les démarches qui passent par le calcul numérique et font appel à de puissantes ressources informatiques prennent une part croissante dans la pratique dite théorique de cette discipline. Les articles déployant essentiellement des déductions formelles se font plus rares. On peut voir là un simple bénéfice de la puissance computationnelle, un complément à la théorie, permettant d'aller vers des solutions plus exactes aux problèmes abordés. Je voudrais pourtant soutenir ici que les rapports de la simulation numérique et de la théorie méritent d'être pensés comme potentiellement problématiques.

\section{Force et prestige de la déduction}

De toutes les sciences de la nature, la physique fut et reste la plus " théorique ", celle qui s'efforce le plus de procéder par déduction, de dégager un corps d'hypothèses aussi compact et cohérent que possible, de formuler des lois fondamentales et de déduire les conséquences de ces lois lorsqu'elle considère les systèmes, réels ou idéaux, où ces lois vont combiner leurs effets. La démarche déductive s'applique aussi bien à l'interprétation qu'à la prédiction. Il en est encore question quand le physicien est conduit à établir que sur tel système, dans telles conditions, la prédiction est entachée d'incertitude, voire impossible. Là encore, jusque dans la détermination de conditions d'instabilité, nous procédons par déduction. Même quand nos démarches heuristiques procèdent par induction et analogies, nous ne sommes satisfaits que lorsque nous avons inséré notre résultat dans un corps formel de propositions. Je laisse de côté les moments, rares, des révolutions épistémologiques, qui fondent un nouveau paradigme.

Le prestige de la théorie lui venait essentiellement de cette double démarche : d'une part faire émerger des lois communes, des structures cachées similaires, voire des isomorphismes rationalisables, d'expériences en apparence hétérogènes ; d'autre part déduire, à partir de principes, des implications non encore mises en évidence. À qui les met en œuvre ou les contemple de l'intérieur, ces pratiques théoriques assurent un vrai plaisir, parfois une émotion esthétique.

Certes la démarche théorique n'est pas la seule pratique du physicien, ni son seul bonheur. Chercher ou inventer des structures nouvelles (comme le font aussi les concepteurs de matériaux et les chimistes), concevoir des modes inédits d'interpellation de la matière, conduire vers l'apparition d'effets nouveaux : ce sont là d'autres versants du plaisir du physicien, où l'imagination tient une autre place que dans les pratiques déductives ou inductives du théoricien. Mais l'imagination du montage nouveau s'élabore encore à partir du corpus théorique déjà disponible. En général nous attendons de l'expérience inédite une confirmation de notre prédiction. Bien sûr, les surprises ont été nombreuses et parfois cruciales dans l'histoire des sciences, quand elles ont invalidé un corpus accepté.
Une prédiction confirmée donne au physicien un sentiment de puissance, un véritable plaisir. Il peut s'agir simplement d'une interprétation élégante faisant appel à un nombre minimal d'éléments logiques. Parfois la prédiction qualitative fait appel à des « ordres de grandeur » qui bornent le domaine du possible. Il faut pourtant noter, chemin faisant, que cette sensibilité aux ordres de grandeur, qui joue un rôle essentiel pour les physiciens, tend à régresser (1). Mais la prédiction nous projette, elle, dans la sphère plus quantitative de la mesure : à quelle distance de la quantité physique observée m'a laissé mon outil de prédiction théorique ? Nous voici dans le domaine du nombre, et la porte est alors ouverte au numérisme et aux simulations.

\section{De la prédiction numérique, illustration de sa fécondité et de ses limites}

Ce qui suit s'applique non seulement à la modeste discipline qui est la mienne, la chimie quantique ou physique moléculaire théorique, mais aussi désormais à certains pans de la physique théorique des solides. Ces deux disciplines ont suivi des chemins assez parallèles. Luttant avec l'équation de Schrödinger, nous avons posé des approximations aussi rationnelles et contrôlables que possible. Nos deux disciplines ont d'abord eu recours à des hamiltoniens simplifiés, ou modèles, et les valeurs attribuées aux opérateurs étaient ajustées sur l'expérience, fonctionnant comme paramètres. On était là dans une forme hybride entre théorie et simulation. La simplicité du hamiltonien permettait encore la production de théorèmes. Mais il était insatisfaisant de changer les valeurs des paramètres quand on passait à une autre observable et en réaction la chimie quantique, qui traite de systèmes électroniques finis, a développé un culte de ce qu'elle appelait $a b$ initio, où le seul empirisme (raisonné) concernait la restriction des espaces de Hilbert dans lequel on travaillerait. Alors, cette discipline a pris sa part dans la recherche de traitements théoriques du problème à $\mathrm{N}$-corps quantique.

Or, la demande de prédiction est énorme, venue de physiciens moléculaires, de spécialistes des matériaux, de chimistes, de biochimistes et de biologistes : "Pourriez-vous nous dire si...?" Ici est intervenue la révolution de la «Théorie de la Fonctionnelle de la Densité » (DFT), qui offrait une sorte de contournement à la résolution de l'équation de Schrödinger dans l'espace des fonctions N-électroniques. La DFT a ses fondements, ses lettres de noblesse, mais aussi ses difficultés. Si elle est devenue si populaire, non seulement en chimie mais en physique des solides, c'est qu'elle a offert un outil de prédiction quantitative économique et relativement efficace. Cette efficacité est passée par l'introduction de fonctionnelles multiples mobilisant la densité locale, puis son gradient, et des ingrédients souvent hétérogènes. Retour fut donc fait en chimie quantique aux méthodes paramétriques ajustées sur l'expérience. Un des codes les plus efficaces mobilise soixante- 
Les traitements plus rigoureux de fonctions d'onde, qui mettent en cuvre des moyens de calcul énormes, renoncent eux aussi très souvent à l'identification des effets vraiment décisifs. On traitera à grands renforts de moyens informatiques des effets qui ne jouent aucun rôle dans la propriété étudiée. deux paramètres (!), ce qui devrait priver ces approches du titre immérité de "first-principle methods". Il est intéressant de noter qu'en physique du solide le prestige de Jacques Friedel, qui incarnait une conception très déductiviste de la science, a retardé l'éclosion en France des approches numériques de type DFT, qui prévalent aujourd'hui lorsqu'une description de champ moyen garde un sens.

En introduisant dans le calcul tant d'informations venues de l'expérience, on entre dans un premier stade de simulation, on quitte la démarche déductive. L'attention se porte alors sur le résultat numérique final. Il devient difficile de procéder à une "décortication " des effets en jeu, ce que permettait une démarche déductive. Disparaissent alors la représentation qualitative des compromis passés entre les différentes composantes, cinétique et potentielle, de l'énergie du système, la traduction des résultats dans une représentation en terme d'effets, la physique "avec les mains ». Mais ce défaut n'est pas l'apanage des approches de type DFT ; les traitements plus rigoureux de fonctions d'onde, qui mettent en œuvre des moyens de calcul énormes, renoncent eux aussi très souvent à l'identification des effets vraiment décisifs. On traitera à grands renforts de moyens informatiques des effets qui ne jouent aucun rôle dans la propriété étudiée. Dans ces démarches qui exhibent la puissance des moyens mobilisés, on a inversé les valeurs traditionnelles d'économie des moyens : il est conseillé d'employer d'emblée les ressources maximales, et seuls comptent en dernière analyse au mieux la précision finale du résultat, au pire le gigantisme du calcul. Autant que la prédiction, ce sont les moyens de la prédiction qui assurent la considération. Ce que les évaluateurs anglo-saxons appellent l'emploi de techniques "state of the art" (que vient faire l'art dans les démonstrations de puissance technique ?) remplace le souci d'économie argumentaire qui fut, depuis le "rasoir d'Occam ", l'un des principes de la démarche scientifique. La suffisance (aux deux sens du terme) peut remplacer la quête de la stricte nécessité ou l'identification des effets déterminants (2).

L'expérience numérique est au contraire particulièrement féconde lorsqu'elle fait surgir une question nouvelle. L'identification des attracteurs étranges en est un bel exemple. Dans certains cas l'exploration algorithmique conduit à des concepts ou des catégories, elle est irremplaçable lorsqu'aucune expérience de laboratoire n'aurait procédé au balayage requis des conditions déterminantes. Et je ne mets pas en cause le recours à la considération sur ordinateur de cas possibles, en nombre fini mais trop grand, qu'ont pu pratiquer des mathématiciens. Ce qui me pose problème chez certains collègues, surtout les jeunes, c'est le recours à l'ordinateur pour obtenir une réponse logiquement accessible, si on ne s'interroge pas, au moins a posteriori, sur la prévisibilité du résultat. 


\section{Vers la pure simulation, sans théorie?}

On voit déjà mises en œuvre, avec un relatif succès, des "learning machines". Celles-ci par exemple s'instruisent sur les propriétés de 5000 molécules et, sur la base de leur seule topologie, sont capables de prédire efficacement les propriétés de 2500 autres molécules (3). Ici on ne fait plus appel à la théorie, on est dans la simulation pure. À partir du graphe moléculaire, sur la base de sa simple topologie (connectivités, nature des atomes, distances entre eux), par des relations phénoménologiques abstruses, on donnera la valeur d'une énergie d'ionisation, donc d'un phénomène intrinsèquement quantique. La "learning machine" ne nous apprend rien, elle donne un nombre. Est-ce là l'avenir ? Pourquoi pas, si notre critère est dans la seule efficacité ?

Si l'on peut injecter soixante-deux paramètres dans une fonctionnelle sophistiquée qui essaye de tenir compte de comportements asymptotiques divers et d'effets variés, une question vient à l'esprit : pourquoi ne pas aller plus loin ? Un simple réseau neuronal, du type de ceux qui parviennent à lire les écritures manuscrites de centaines de personnes, voire de reconnaître les préférences sexuelles des sujets (4), ne serait-il pas aussi prédictif que des méthodes qui se réferent encore à un formalisme quantique ? Il s'agirait de l'usage de câblages aléatoires et de processus d'apprentissage, donc d'une prédiction sans théorie ni relations empiriques. On entre là dans une efficacité sans théorie. Ce type de simulation peut congédier la démarche théorique.

\section{Qu'entend-on par « comprendre » ?}

Mais l'accent que je mets sur le souci de compréhension est peut-être ringard. Jusqu'où fonctionne notre exigence de compréhension ? Ne nous abandonnons-nous pas chaque jour à des dispositifs techniques que nous ne comprenons pas et ne cherchons pas à comprendre ? Le philosophe Günther Anders, dans L'obsolescence de l'homme (5), pensait que la condition de l'homme moderne était marquée par l'humiliation que lui inflige la machine, désormais plus performante que lui. Il ne semble pas que ce diagnostic soit juste : nous profitons de machines que d'autres ont conçues et mises à notre service et dont nous ne connaissons pas le fonctionnement. Jusqu'où les générations qui viennent iront-elles dans ce sens ? Qui, déjà, se rappelle les règles d'extraction des racines carrées ? Les enfants du milieu de ce siècle poseront-ils encore des divisions?

Certains diront donc qu'il serait pertinent de laisser à la simulation ce qu'elle peut traiter, et d'abandonner les rationalisations de comportements que la simulation numérique peut (re)produire. Dans ce scénario nous devrions centrer nos efforts scientifiques sur des enjeux plus sérieux, ceux que la seule simulation ne peut pas aborder, concentrer la théorie sur les constructions fondamentales, principielles. J'aurais pourtant de la peine, je l'avoue, à renoncer aux explications simples et fondées qui me permettent d'interpréter, (presque) sans calcul, tel phénomène ou telle propriété un peu inattendue de tel édifice moléculaire, dès lors qu'une simulation numérique apporterait une réponse plus quantitative. Car, de façon plus large, l'exercice de la raison à des problèmes modestes représente un élément précieux et socialement crucial de notre culture.
J'aurais de la peine à renoncer aux explications simples et fondées qui me permettent d'interpréter, (presque) sans calcul, tel phénomène ou telle propriété un peu inattendue de tel édifice moléculaire [...] Car l'exercice de la raison à des problèmes modestes représente un élément précieux et socialement crucial de notre culture.

\section{D'autres invasions du numérisme}

Nous retrouvons donc, avec une évidence accrue, la pertinence de l'affirmation de René Thom : "prédire n'est pas comprendre ". Mais qui désire encore comprendre, comment maintenir cette envie, et la satisfaction profonde qu'assurait la démarche déductive, le frisson que nous avons éprouvé, étudiants, en voyant les lois de Maxwell fondre en un seul corps trois domaines auparavant disjoints de la physique ? Plus tard, devant la beauté du groupe de renormalisation de Wilson ? Le statut, à la fois réel et symbolique, de la théorie est sans doute en question. Pas seulement en physique, mais aussi dans la compréhension que nous pouvons avoir des sociétés dans lesquelles nous sommes immergés. Non, le "Big Data" ne remplacera pas les modèles interprétatifs, certes imparfaits, que nous proposent les sciences humaines, par des corrélations étayées sur des millions de données. La mise en évidence de corrélations est un moment utile, mais une corrélation n'établit pas un lien de causalité. Rappelons à nos élèves que la théorie est autre chose qu'un faisceau de corrélations, qu'elle exige la construction d'une architecture interprétative. Mais la construction théorique exige de son auteur (et de ceux qui l'assimilent) une concentration intellectuelle qui n'obéit pas à la recherche de réponses et de satisfactions immédiates, et cette démarche ne garantit pas nécessairement un "h-index" important ni des financements sur projet. Car le temps de la théorie n'est pas celui, plus court, de la simulation, et la simulation paye davantage que la théorie en matière de publications, donc de carrière. Là encore nous attribuons à la quantité une excessive vertu d'objectivité.

Jean-Paul Malrieu (jean-paul.malrieu@irsamc.ups-tlse.fr) Laboratoire de Chimie et Physique Quantiques, Université Paul Sabatier, 118 route de Narbonne, 31062 Toulouse Cedex 09

(1) Ce texte part d'une brève intervention lors d'une rencontre célébrant le départ à la retraite de Jacques Vigué, bien connu pour sa maitrise des ordres de grandeur et leur invocation pertinente. On trouverait d'autres exemples de déductions fondées sur des ordres de grandeur chez Jean-Marc Lévy-Leblond ou Pierre-Gilles de Gennes.

(2) On notera que malheureusement la démarche qui s'appuierait sur des calculs complexes pour fonder des hamiltoniens modèles simplifiés, qu'on pourrait ensuite manipuler sans ordinateur selon des procédures déductibles, tend elle aussi à régresser.

(3) Voir par exemple G. Montavon et al., New J. Phys. 15 (2013) 095003, ou K. Yao et al., J. Phys. Chem. Lett. 6 (2015) 2326. L'auteur remercie Roald Hoffman pour lui avoir indiqué ces travaux et ceux de la référence suivante.

(4) M. Kozinski, Y. Wang. J. Pers. Soc. Psych. (septembre 2017)

(5) G. Anders, L'obsolescence de l'homme, Éditions Ivrea (2002) 